\title{
HIGH STRAIN-RATE COMPRESSIVE TESTING OF FILLING MATERIALS FOR INTER-PENETRATING PHASE COMPOSITES
}

\author{
Tomáš Doktor ${ }^{a, *}$, Tomáš Fíla $^{a}$, Petr Zlámal $^{a, b}$, DAniel Kytýř ${ }^{a, b}$, \\ ONDŘEJ JIROUŠEK ${ }^{a}$ \\ ${ }^{a}$ Czech Technical University in Prague, Faculty of Transportation Sciences, Konviktská 20, 11000 Prague, Czech \\ Republic \\ ${ }^{b}$ Academy of Sciences of the Czech Republic, Institute of Theoretical and Applied Mechanics, Prosecká 76, \\ 19000 Prague, Czech Republic \\ * corresponding author: doktor@fd.cvut.cz
}

\begin{abstract}
In this study behavior of the selected types of filling material for the inter-penetrating phase composites was tested in compressive loading mode at low and high strain-rates. Three types of the filling material were tested, (i) ordnance gelatin, (ii) low expansion polyurethane foam, and (iii) polyurethane putty. To evaluate their impact energy absorption bulk samples of the selected materials were tested in compression loading mode at strain-rates $1000 \mathrm{~s}^{-1}$ to $4000 \mathrm{~s}^{-1}$. The high strain-rate compressive loading was provided by Split Hopkinson Pressure Bar (SHPB) which was equipped with PMMA bars to enable testing of cellular materials with low mechanical impedance. Based on the comparative measurement response to compression at both low and high strain-rates was analysed. The results show a significant strain-rate sensitivity of the ordnance gelatin and of the polyurethane putty, while strain-rate effect in the polyurethane foam was not observed.
\end{abstract}

KEYWORDS: Interpenetration phase composite, SHPB, impact protection filling materials, strain-rate sensitivity.

\section{INTRODUCTION}

In recent decades cellular materials, both metallic and polymeric, are of interest in engineering design fields due to their lightweight nature and possibilities for structural and topological optimisation. Among such cellular materials, metal foams [1, additively manufactured periodic structures and auxetics (materials with negative Poisson's ratio) may serve in impact energy absorption applications. For this purpose, materials exhibiting plateau region with increasing of strain keeping constant or only slightly increasing stress up to considerably high strain are required. A high potential for impact energy protection pose materials with strain-rate sensitive behaviour which increase their stress response with increasing strain-rate. Such materials may be identified among closed cell metal foams, where strain-rate sensitive response is induced by air flow trough the cell walls during their failure [2, or coated open cell metal foams in which the strainrate sensitivity is caused by micro-inertia effects of the struts 3 . Another way to enhance cellular metals' impact energy absorption properties is to equip the porous structure with a suitable filling material to form inter-penetrating phase composite (IPC). The filling has to provide a good strain energy absorption with acceptable increase of the overall density of such IPC. In this study behavior of selected types of filling material were tested in compressive loading mode at quasi-static condition and using Hopkinson Pressure Bar (SHPB) at high strain-rates.

\section{MATERIALS AND METHODS}

\subsection{SPECIMEN PREPARATION}

Three groups of specimens were prepared from three different polymeric materials: (i) polyurethane foam, (ii) polyurethane putty and (iii) ordnance gelatin. Cylindrical shape and dimensions (diameter $18 \mathrm{~mm}$ and length $5 \mathrm{~mm}$ ) of the sample were selected with respect to bars' diameter in SHPB apparatus. Both polyurethane foam and polyurethane putty were commercially available single component substances which cured in several hours under ambient conditions. Due to its expandible nature, polyurethane foam was poured into a larger mold and subsequently cut by an in house metalic hollow drill to the desired shape. Finally, length of the cylinder was finalized by sharp blade. The polyurethane putty was prepared in a confined mold, and after curing process, the cylinders shortened by sharp blade. The ordnance gelatin samples was prepared according to recipe published by Jussila [4. The liquid mixture was poured in a shallow mold to achieve uniform height and after curing cylindres were cut by a hollow drill. The same shape and dimensions of the specimens were used for both quasi-static and dynamic tests.

\subsection{QuAsi-STATIC TESTS}

To obtain reference stress-strain curves for evaluation of behaviour under higher strain-rates, a series of quasi-static tests of all three materials were performed. For the quasi-static tests, a custom uniaxial loading 


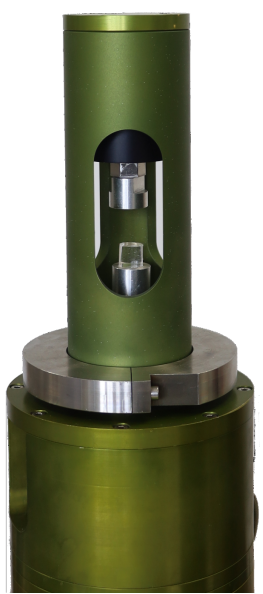

Figure 1. Experimental setup used for quasi-static tests.

frame [5] with loading capacity $3 \mathrm{kN}$ was used. The loading setup was instrumented by a load-cell U9b (Hottinger Baldwin Messtechnik, Germany) with loading capacity $1 \mathrm{kN}$. Loading was provided by a stepper motor attached to a harmonic drive and controlled by an in-house control software based on LinuxCNC interface [6]. The loading setup for the quasi-static tests is depicted in Figure 1] A custom control unit for both loading axis control and load-cell readout was used [7].

The displacement controlled test was performed up to overall strain of $50 \%$ with loading rate of $4 \mu \mathrm{m} . \mathrm{s}^{-1}$ corresponding to strain-rate appox. $5 \cdot 10^{-3} \mathrm{~s}^{-1}$.

\subsection{High STRAin-RATE TESTS}

For the high strain-rate tests, an in-house SHPB, developed at CTU in Prague [8] was used. The apparatus is based on Kosky setup, which was adapted for testing of materials with low mechanical impedance. The striker, incident and transmission bars were maufactured from Poly Methyl-metacrylate (PMMA). Diameter of the bars was $20 \mathrm{~mm}$, lengths of striker, incident and transmission bars were $190 \mathrm{~mm}, 1600 \mathrm{~mm}$ and $1600 \mathrm{~mm}$, respectively. The bars were supported by 8 low-friction polymer-liner slide bearings with aluminum housing (DrylinFJUM housing, IGUS, Germany). The striker bar was accelerated by air gun with maximum pressure 16 bar with fast release solenoid valve (366531, Parker, USA).

Different levels of pressure were used to accelerate the striker in range from 0.5 bar to 3.0 bar. Based on the preliminary results, pressure levels of 0.5 bar, 1.0 bar and 1.5 bar were selected for testing of polyurethane foam and polyurethane putty and pressure levels of 1.0 bar, 2.0 bar and 3.0 bar were used for the samples of ordnance gelatin.

The incident bar was instrumented with 3 measurement points using strain-gauges to take the wave propagation phenomena in visco-elastic medium into account. The transmission bar was instrumented with single measurement point. The foil strain-gauges (3/120 LY61, Hottinger Baldwin Messtechnik, Germany) were applied on each measurement point using a single component low-viscosity cyanoacrylate adhesive (Cyberbond 2003, Cyberbond Europe GmbH, Germany). The strain-gauges were connected in Wheastone half-bridge arrangement to compensate the possible minor bending of the bars during the latter stage of the experiment. The signals were amplified by an active differential low-noise amplifier (EL-LNA-2, Elsys AG, Switzerland) and recorded by high speed 16bit digitizer (PCI-9826H, ADLINK Technology, Inc., Taiwan) with maximum sample rate of $20 \mathrm{MHz}$. Due to a limited capacity of the digitizer, precise triggering of the measurement was required. The trigger was implemented by a pair of laser through-beam photoelectric sensor (FS/FE10-RL-PS-E4, Sensopart, Germany) which were installed on the barrel.

Moreover a high speed camera (FASTCAM SA5, Photron, Japan) was used to capture the loading scene at frame rate $124000 \mathrm{fps}$ which enabled visual inspection of the deformation behavior of the tested specimens. The SHPB setup is shown in Figure 2.

\section{Results}

\subsection{Quasi-STATIC TESTS}

Stress-strain curves of all tested specimens were calculated using the data recorded during the experiments. In the mechanical response of the polyurethane putty, progressive densification occured during the entire compressive loading. In the response of the polyurethane foam, the elastic region followed by the plateau and the densification was observed. Response of the ordnance gelatin exhibits steady densification. The stress-strain curves are shown in Figure 3 .

\subsection{High STRAin-RATE TESTS}

In the high strain-rate tests, stress-strain curves were derived using the strain-gauges signals. From known wave propagation velocity in the bars' material and measured strain, strain rate and strain was calculated based on the wave propagation theory in onedimension. Then, from the strain and known crosssection area of both bars and sample and elastic modulus of the bars, stress was derived. To check validity of the performed tests, force equilibrium (convergence between input and output force at transmission bar) was evaluated. Example of the equilibrium evaluation, where force calculated at incident and transmission bar is plotted over time, is presented in Figure 4

In total, 5 tests were performed for every pressure level in each sample group. The stress-strain diagrams for the group of the polyurethane putty, the polyurethane foam and the ordnance gelatin, together with strain-rate-strain curves, are presented in Figures 5.7. Note, that strain-rate of the experiments varied from $1000 \mathrm{~s}^{-1}$ to $4000 \mathrm{~s}^{-1}$. 


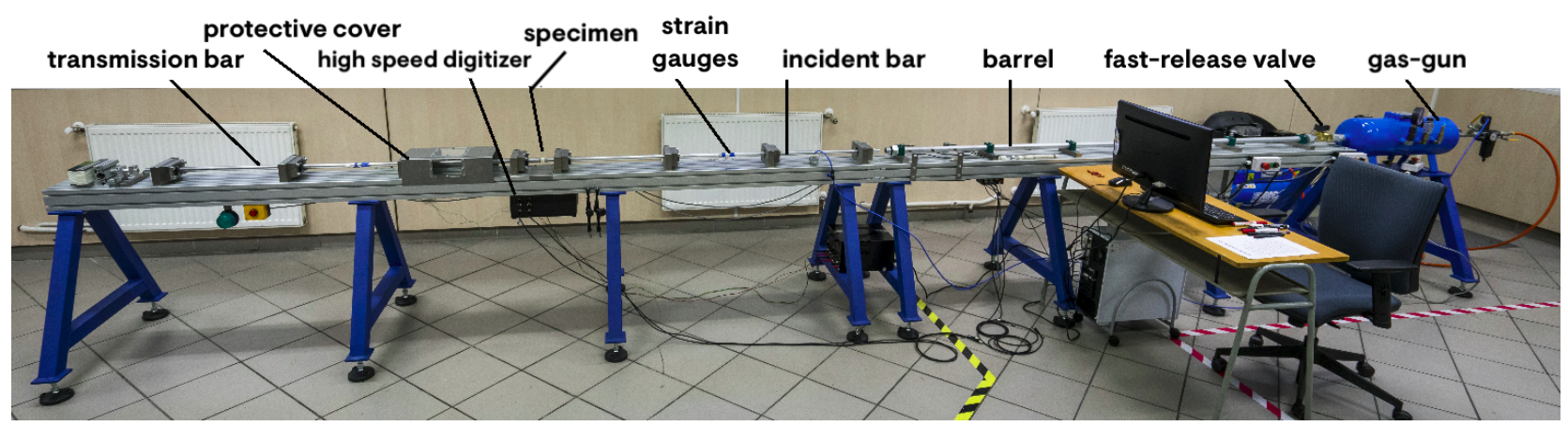

Figure 2. SHPB setup.
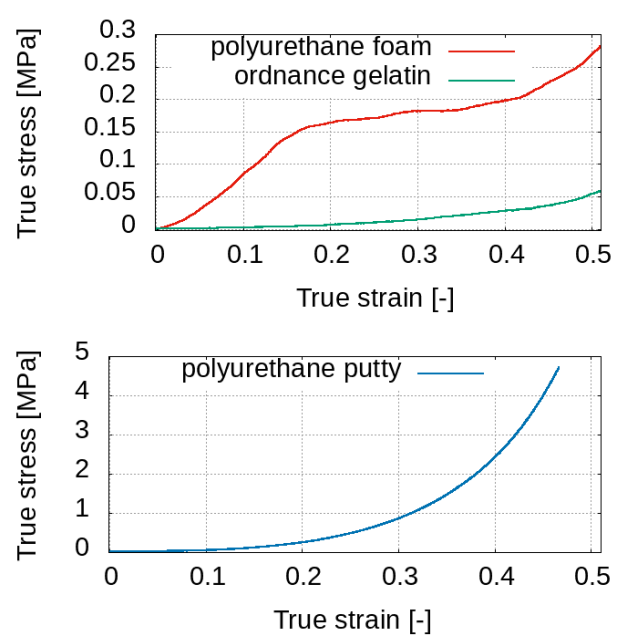

FigURE 3. Stress-strain curves under quasi-static compression.

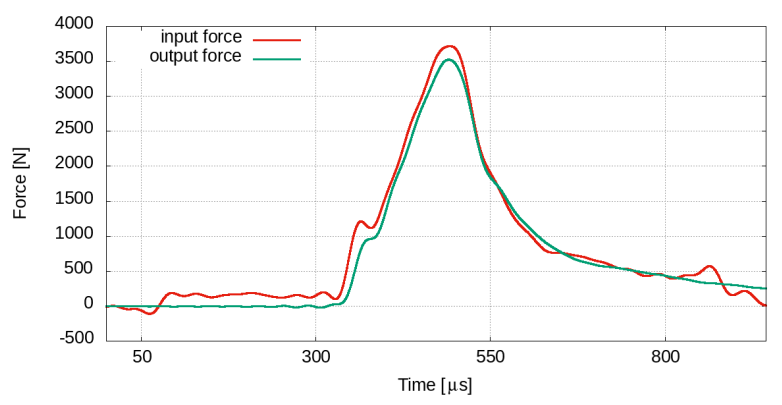

FIGURE 4. Force equilibrium diagram for polyurethane putty specimen tested at 1.5 bar.

The characteristics of the plastic failure were evaluated using the high-speed camera images. Comparison between the intact and the damaged state of the selected specimens in all three groups are shown in Figures 8 to 10 . The specimens of the polyurethane foam exhibit no significant radial expansion during the plateau region, which corresponds to its porous nature and crushing of the pores during the plateau. On the other hand, radial expansion of the polyurethane putty specimens is clearly detectable during the entire deformation evolution, which corresponds to the steady increase of stress without significant plateau. The specimens of the ordnance gelatin exhibit slight

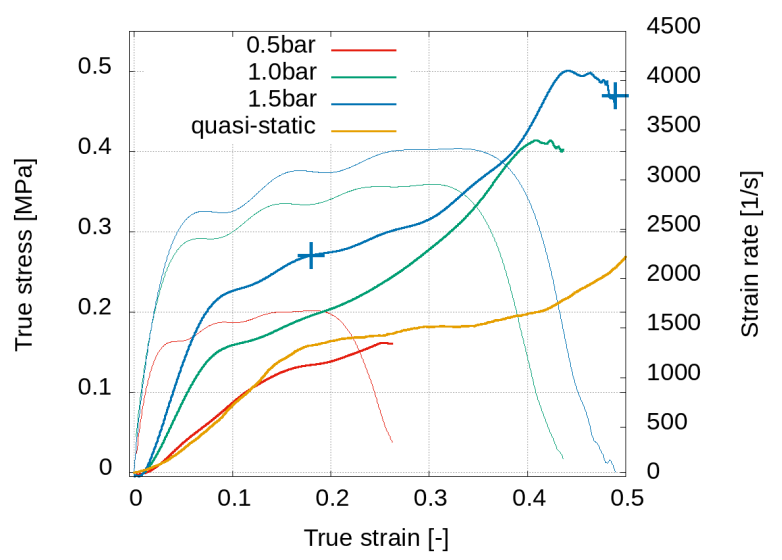

FIGURE 5. Stress-strain curves and strain-rate evolution for group of polyurethane foam specimens.

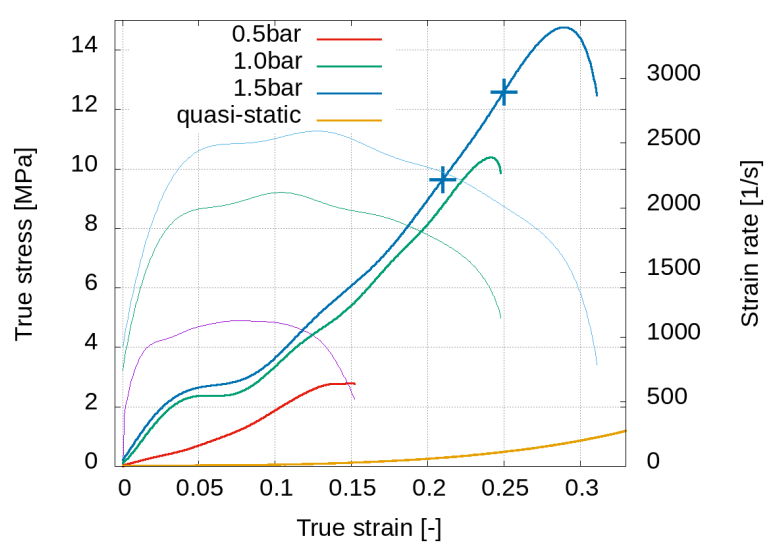

FiguRE 6. Stress-strain curves and strain-rate evolution for group of polyurethane putty specimens.

radial expansion during the initial phase of tests and subsequently fail may be identified at the surface, development of which occures in the plateau phase of the stress-strain curves.

\section{Conclusions}

Based on the comparative measurement, the response to compressive loading of three types of filling material was evaluated. The obtained stress-strain curves were analyzed together with the sets of images of intact and damaged specimen during high strain-rate loading 


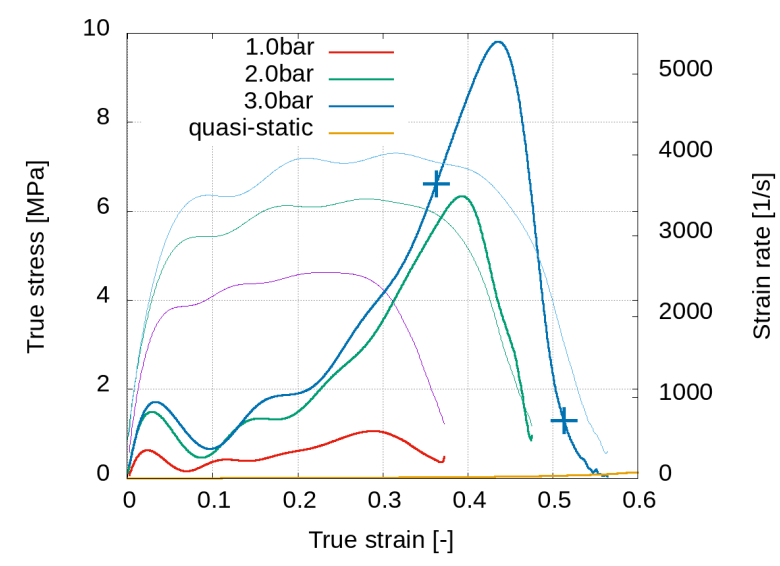

FiguRE 7. Stress-strain curves and strain-rate evolution for group of ordnance gelatin specimens.
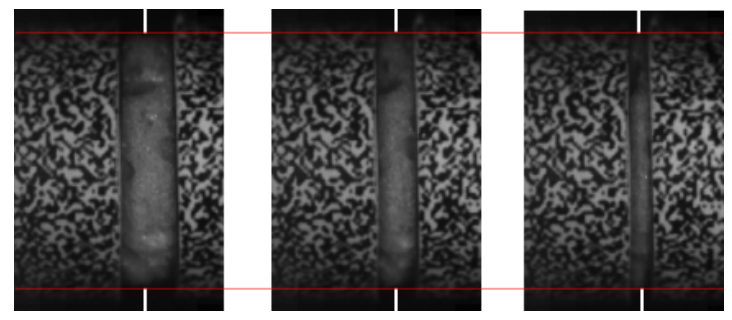

Figure 8. Comparison of intact and deformed state of polyurethane foam specimen.

and the behaviour corresponds to the measured curves. The results show a significant strain-rate sensitivity of the ordnance gelatin and of the polyurethane putty. Strain-rate effects in polyurethane foam occured starting at strain rates higher than $1500 \mathrm{~s}^{-1}$. However, this fact might be influenced by the limited dimensions of the specimens required for the SHPB tests which did not allow to test the representative volume element in this case.

\section{ACKNOWLEDGEMENTS}

The research has been supported by the Czech Science Foundation (project No. 19-23675S) and by Operational Programme Research, Development and Education in project Engineering applications of microworld physics (CZ.02.1.01/0.0/0.0/16_019/0000766).

\section{REFERENCES}

[1] P. Qiao, M. Yang, F. Bobaru. Impact mechanics and high-energy absorbing materials: Review. Journal of Aerospace Engineering 21(4):235-248, 2008. DOI:10.1061/(ASCE)0893-1321(2008)21:4(235)

[2] K. Dannemann, J. Lankford Jr. High strain rate compression of closed-cell aluminum foams. Materials Science and Engineering A 293(1):157-164, 2000. DOI:10.1016/S0921-5093(00)01219-3

[3] A. Jung, S. Diebels. Synthesis and mechanical properties of novel ni/pu hybrid foams: A new economic composite material for energy absorbers. Advanced Engineering Materials 18(4):532-541, 2016. DOI:10.1002/adem.201500405.
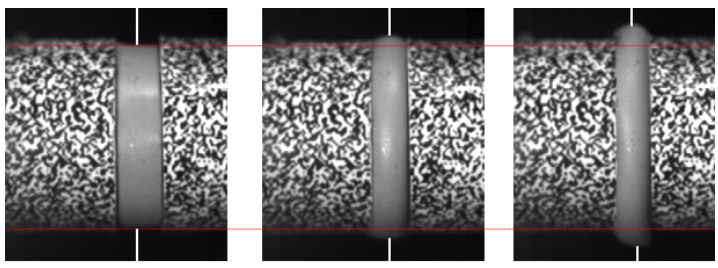

Figure 9. Comparison of intact and deformed state of polyurethane putty specimen.
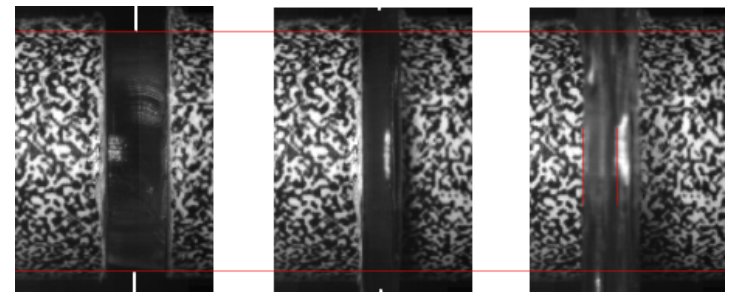

Figure 10. Comparison of intact and deformed state of ordnance gelatin specimen. Middle: white bars highlight the radial expansion. Right: Overlap of the gelatin over the transmission bar highlighted by red lines.

[4] J. Jussila. Preparing ballistic gelatine - review and proposal for a standard method. Forensic Science International 141(2-3):91-98, 2004. DOI:10.1016/j.forsciint.2003.11.036.

[5] T. Fila, J. Sleichrt, D. Kytyr, et al. Deformation analysis of the spongious sample in simulated physiological conditions based on in-situ compression, $4 \mathrm{~d}$ computed tomography and fast readout detector. Journal of Instrumentation 13(11), 2018. DOI:10.1088/1748-0221/13/11/C11021

[6] V. Rada, T. Fila, P. Zlamal, et al. Multi-channel control system for in-situ laboratory loading devices. Acta Polytechnica Proceedings 18:15-19, 2018. DOI:10.14311/APP.2018.18.0015.

[7] D. Kytyr, N. Krcmarova, J. Sleichrt, et al. Deformation response of gellan gum based bone scaffold subjected to uniaxial quasi-static loading. Acta Polytechnica 57(1):14-21, 2017. DOI:10.14311/AP.2017.57.0014

[8] T. Fila, P. Zlamal, O. Jirousek, et al. Impact testing of polymer-filled auxetics using splithopkinson pressure bar. Advanced Engineering Materials 19(10):1-13, 2017. DOI:10.1002/adem.201700076 